\title{
Small RNA Sequencing and Bioinformatics Analysis of RAW264.7-Derived Exosomes after Mycobacterium Bovis Bacillus Calmette-Guérin Infection
}

\section{Xuehua Zhan}

Ningxia Medical University

Wenqi Yuan

Ningxia Medical University

Yueyong Zhou

Ningxia Medical University

Rong Ma

Ningxia Medical University General Hospital

Zhaohui Ge ( $\nabla$ myovid@126.com )

Ningxia Medical University General Hospital

\section{Research Article}

Keywords: Exosomal miRNA, Tuberculosis, BCG, macrophages, apoptosis, RNA-seq

Posted Date: February 18th, 2022

DOI: https://doi.org/10.21203/rs.3.rs-1297482/v1

License: (c) (i) This work is licensed under a Creative Commons Attribution 4.0 International License.

Read Full License 


\section{Abstract}

Background: The mechanisms through which Mycobacterium tuberculosis evades immune surveillance during tuberculosis (TB) infection remain complex. Previous studies have found that Mycobacteria can manipulate the miRNAs of host cells to promote their survival during host-pathogen interactions, and most of these effects occur at the cellular miRNA level. We at-tempted to investigate the possible related mechanisms at the exosomal miRNA level.

Results: High-throughput sequencing revealed that Bacillus Calmette-Guérin (BCG) infection could alter the composition of the macrophage exosome content, and the expression levels of miRNAs in exosomes derived from the cell culture media of macrophages showed significant differences be-tween the BCGinfected and noninfected groups. Compared with the non-infection group, 20 exosomal miRNAs were upregulated and 7 exosomal miRNAs were down-regulated in the infection group $(p<0.05)$, of which mmumiR-27b-3p, mmu-miR-93-5p, mmu-miR-25-3p, mmu-miR-1198-5p, mmu-let-7c-5p and let-7a-5p were significantly up-regulated. A bioinformatic analysis indicated that these differentially expressed exosomal miRNAs were involved in multiple biological processes and pathways. The target genes of top six miRNAs in up-regulated groups were positively correlated with the regulation of apoptosis.

Conclusions: Our study provides relatively strong evidence for unravelling the complex pathophysiological processes of TB and the mechanisms of mycobacterial immune escape based on differential exosomal miRNA expression in host macrophages. Additionally, these exosomal miRNAs may serve as potential immunotherapy targets for the effective control of TB infection, but this probability requires further study.

\section{Background}

Tuberculosis (TB), mainly caused by the Mycobacterium tuberculosis complex (MTBC), is the uppermost catastrophic infectious disease that threatens human health and safety. Although the number of newly diagnosed TB decreased from 7.1 million to 5.8 million between 2019 and 2020, there remains 1.3 million HIV-negative individuals and an additional 214,000 HIV-positive individuals died of TB in 2020(1).

Macrophages act as a central role in the innate immunity process because these cells constitute the initial cellular niche for mycobacterial pathogenesis during infection. Through intricate pathogen recognition approaches, macrophages can orchestrate multiple signalling cascades to trigger various innate immune defences, include phagocytosis, apoptosis, autophagy and inflammasome activation, to eliminate Mtb (2). Moreover, Mtb has evolved a series of intelligent strategies to hijack host cell miRNAs to subvert the abovementioned functions and achieve immune evasion (3). Numerous emerging studies have revealed that miRNAs can manipulate host macrophage defence against Mtb infection $(4,6)$, but a detailed and comprehensive understanding of the mechanisms remains lacking.

MicroRNAs (miRNAs) are widely conserved small noncoding RNAs with a length of 18-24 nucleotides that display an indispensable role in many physiological and pathological aspects through the 
posttranslational regulation of target gene expression (7). Exosomes are bioactive lipid bilayerencapsulated extracellular vesicles with a diameter of 30-150 nm, derived from virtually all cell types. Exosomes circulate in the extracellular environment and are capable of shuttling various vital molecule elements, for instance, nucleic acids (including miRNAs), proteins and lipids (8).

The majority of previous studies focused on differential expression analysis and the functions of miRNAs at the cellular level. However, we attempted to investigate the specific mechanism of immune escape adopted by Mtb based on exosomal miRNA levels by small RNA transcriptome high-throughput sequencing and bioinformatics.

\section{Materials And Methods}

\section{Cell and BCG culture}

RAW264.7 cells preserved in our laboratory at a low passage number were cultured in a T-75 flask (Corning, USA) with Dulbecco's modified Eagle's medium (DMEM; Gibco, USA) containing 1\% L-glutamine, $1 \mathrm{mM}$ sodium pyruvate, $10 \%$ foetal bovine serum (FBS; Gibco, USA) and 1\% penicillin-streptomycin at $37^{\circ} \mathrm{C}$ in an $5 \% \mathrm{CO}_{2}$ atmosphere.

Mycobacterium bovis Bacillus Calmette-Guérin (BCG) St. Pasteur 1173P2 strain was frozen and stored in our laboratory. The BCG bacilli were grown in Mycobacterium complete medium (Gene-Optimal, Shanghai, China) at $37^{\circ} \mathrm{C}$, which was achieved to the logarithmic phase of growth after 10 to 12 days of culture and then aliquoted. A turbidimetric assay was conducted with a spectrophotometer (Infinite 200 PRO, TECAN, Switzerland) to measure the optical density (OD) of the bacteria at a wavelength of $600 \mathrm{~nm}$. Once the OD value of the culture medium reached approximately $0.9-1.0$, the BCG bacilli were harvested by centrifugation for 10 minutes at $4500 \mathrm{rpm}$ and then resuspended in DMEM without antibiotics as previously described (9).

\section{Infection assay}

The cells were planted into twelve T-75 flasks $\left(5 \times 10^{6}\right.$ cells/flask) prior to the day of infection. All the cells were classified into a non-infection group (Group A) and an infection group (Group B), and each group was analysed in triplicate. On the day of infection, the macrophage monolayers were gently washed three times with phosphate buffered saline (PBS; BI, Israel) at room temperature. Set multiplicity of infection to 10 . The cells in Group B were stimulated with BCG and incubated at $37^{\circ} \mathrm{C}$ in an incubator (Thermal, USA) with $5 \% \mathrm{CO}_{2}$ for 4 hours, whereas PBS was added to the cells in Group A as a control. After incubation, the supernatant culture medium was replaced, and the adherent monolayers were tenderly washed with warm $\left(37^{\circ} \mathrm{C}\right) \mathrm{PBS}(\mathrm{BI}$, Israel) three times to exclude extracellular bacteria (10). Added fresh DMEM (Gibco, USA) with 10\% exosome-depleted FBS (VivaCell Biosciences, China) and continue to culture the cells for 72 hours. All of the cell nutrient media were gathered and then centrifuged 10 minutes $(3000 \times \mathrm{g})$ to eliminate cell debris and then passed through a $0.22-\mu \mathrm{m}$ filter unit. At least $50 \mathrm{ml}$ 
of the cell culture supernatants from each replicate were needed to acquire sufficient amounts of exosomes for the downstream experiments.

\section{Exosome characterization and exosomal RNA preparation}

The Cell Culture Media Exosome Purification Kits (Norgen Biotek, Canada) were used to extract exosomes from the above-described cell culture medium in accordance with the instructions. Concisely, the required cell culture supernatants were gathered, transferred into a conical tube, and centrifuged $(200 \times \mathrm{g}, 15$ minutes) to discard cells and debris, and the cell-free medium was then transferred to a fresh conical tube for the exosome purification. ExoC buffer and Slurry E were added separately to fresh media at different proportions according to the instructions, vortexed for 10 seconds at first and then incubated for 10 minutes at room temperature. The media were remixed and centrifuged for 2 minutes at 2,000 rpm. Subsequently, the ExoR buffer $(600 \mu \mathrm{l})$ were applied to the slurry pellet, and the pellet was incubated for a quarter at room temperature and centrifuged at $500 \mathrm{rpm}$ for 2 minutes. The supernatants were then moved into a fresh Mini Filter Spin column and centrifuged at $6,000 \mathrm{rpm}$ for 1 minute. PBS was chosen to suspend the sedimentation and preserved it at $-20{ }^{\circ} \mathrm{C}$. Transmission electron microscopy (TEM) (Hitachi, HT-7700, Japan) and nano flow cytometry (N30E, NanoFCM, Xiamen, China) were used to characterize the purified exosomes. Total exosomal RNA isolation and extraction were performed using an exosomal RNA isolation kit (Norgen Biotek, Canada) according to the instructions. The RNA concentration and purity were detected by Agilent 2100 Bioanalyzer (Agilent Technologies, USA).

\section{Small RNA library construction and miRNA sequencing}

The construction and sequencing of Small RNA library comply with the standard operating program provided by Illumina. A TruSeq Small RNA Sample Prep Kit (Illumina, San Diego, CA, USA) was utilized to prepare the library. After that, an Illumina HiSeq 2500 instrument was chosen to sequence the constructed library. An in-house program, ACGT101-miR (LC Sciences, Houston, TX, USA) was applied to subject the raw reads and to delete junk sequences, adapter dimers, low complexity reads and duplicates. Finally, 18 26 nucleotides distinctive sequences were mapped to precursors of specific-species in miRbase 22.0 using the Basic Local Alignment Search Tool (BLAST) to discriminate against known and novel miRNAs derived from $3 p$ and $5 p$.

\section{Analysis of the differential expression of miRNAs}

The differential expression of miRNA was analysed by the Student 's $t$ test. Based on standardized deep sequencing, the differential expression genes were screened by $p \leq 0.05$. Differential miRNA cluster analysis was selected to determine the cluster pattern of miRNA regulation under diverse experimental conditions. According to the statistical similarity of the miRNA expression profiles of the samples, miRNA cluster analysis was applied to intuitively display the distribution of miRNAs in different samples using the log10-transformed normalized values in the heatmap. An advanced volcano map was plotted to manifest the overall distribution and expression levels of these miRNAs. 


\section{Prediction of the target genes}

Prediction of the target genes of differentially expressed miRNAs was accomplished by TargetScan (v5.0) and Miranda (v3.3a). The overlap between the data predicted by the two algorithms was obtained. Targeted genes with a context score less than 50 obtained with the TargetScan algorithm were filtered, and those with a maximum free energy (maximal energy) greater than -10 obtained with the Miranda algorithm were removed. The intersections between these two software programs were considered the ultimate target genes and their Gene Ontology (GO) and Kyoto Encyclopedia of Genes and Genomes (KEGG) information of these miRNAs was annotated.

\section{Target genes enrichment}

The enrichment analysis mainly consisted of two evaluations: GO and KEGG pathway functional annotation. First, count the number of genes corresponding to each function or pathway annotation for all selected miRNAs, and then perform hypergeometric test to determine the number of genes corresponding to different terms in the annotation library. The threshold was defined as $p$-value $\leq 0.05$, and the genes satisfying this condition were considered as dominant and endogenous genes. The GO and KEGG functional enrichment analysis of the screened target genes were accomplished by using the OmicStudio tools (https://www.omicstudio.cn/too).

\section{Visualization of the KEGG analysis results}

The target genes which involved in cell growth and death regulation were selected and used for the subsequent data visualization analysis with Metascape (https://metascape.org). Based on the membership similarities, those $p$ value less than 0.01 , enrichment factor more than 1.5 and a minimum count of 3 terms were gathered and classified into clusters. In addition, a protein-protein interaction (PPI) enrichment analysis was accomplished using the following databases: STRING (11), BioGRID (12), OmniPath (13), and InWeb_IM (13). The obtained network is composed of proteins subsets that physically interacted with at least one other member on the list. The Molecular Complex Detection (MCODE) algorithm is used to classify dense network elements if the network contains $3 \sim 500$ proteins (14). Network visualization was performed using Cytoscape (v3.1.2) (15).

\section{Verification of exosomal miRNAs by qRT-PCR}

The experimental grouping, cell culture and BCG infection procedures were the same as those previously described. Each group consisted of three biological replicates, the exosomes were isolated by ultracentrifugation (UC), and the total RNA was extracted from the exosomes. A ReverTra Ace qPCR RT Kit (TOYOBO, Japan) was used for the CDNA reverse transcription according to the instructions provided by the manufacturer. A stem-loop method was selected for the first-strand cDNA synthesis, and the primer sequences are presented in Table 1. Quantitative real-time polymerase chain reaction (qRTPCR) was completed using a LightCycler 96 instrument (Roche, Switzerland) with SYBR Green RealTime PCR Master Mix (TOYOBO, Japan). A relative quantitative analysis of the miRNAs was carried out 
by $2^{-\triangle \Delta C T}$ method, and standardization was performed with respect to $U 6$ as the reference gene. Three biological replicates of each group were included, and each sample was analysed in three independent repeated trials.

\section{Statistical analyses}

The statistical analysis was performed using GraphPad Prism 8.4 independent sample $t$ - test. Statistical significance was assumed at $p \llbracket 0.05$.

\section{Results}

\section{Exosome characterization and exosomal RNA preparation}

The supernatants from the culture media from the two groups were collected and used for the exosome isolation. The exosome morphology was evaluated by TEM. NanoFCM was used for the detection of exosome transmembrane proteins (CD9 and CD81) and to determine the particle concentration and size distribution.

As shown in Fig. 1, the typical morphology of exosomes can be observed by TEM; the exosome transmembrane proteins CD9 and CD91 showed positive expression in $14.1 \%$ and $8.7 \%$ of the exosomes, respectively; the average concentration was $7.45 \times 10^{9}$ particles/ml, and the particle size was mainly distributed in the $60-80-\mathrm{nm}$ range (Fig. 2). The concentration of exosomal RNA extracted from the exosomes in each sample varied from 0.0021 to $0.0039 \mu \mathrm{g} / \mu \mathrm{l}$. Quality control and an RIN evaluation were performed, and the obtained values indicated that the samples met the standards for the subsequent sequencing analysis.

\section{Analysis of the differential expression of miRNAs}

In total, 1853 miRNAs were detected, and 506 miRNAs overlapped between the infection group and noninfection group (Fig. 3). A differential miRNA cluster analysis intuitively displayed the specific expression profiles of the miRNAs in the different experimental treatments (Fig. 4), and the advanced volcano map displays the significant differential expression of multiple miRNAs (Fig. 5). The data showed that, compared with the non-infection group, there were 20 miRNAs upregulated and 7 miRNAs downregulated significantly in the infection group. The expression levels and the upor downregulation relationships are presented in Table 2. Among the detected miRNAs, mmu-miR-27b-3p, $-93-5 p,-25-3 p,-1198-5 p$, -let-7c-5p and -let-7a-5p were expressed at relatively high levels. Target gene prediction and GO and KEGG enrichment analyses of these six significantly differentially expressed miRNAs were subsequently performed.

Finally, a number of 5643 target genes were predicted by using TargetScan (v5.0) and miRanda (v3.3a) software. GO and KEGG enrichment analysis of the screened target genes was then performed using the 
OmicStudio tools (https://www.omicstudio.cn/tool), and in total, 5576 target genes were effectively enriched.

\section{GO enrichment analysis}

The biological process (BP) enrichment analysis revealed that $92.88 \%, 71.98 \%, 45.57 \%, 43.33 \%, 43.33 \%$, $34.36 \%, 30.28 \%, 27.73 \%, 25.39 \%$ and $22.84 \%$ of the target genes mainly participated in signal transduction, the $\mathrm{G}$ protein-coupled receptor signalling pathway, response to stimulation, the detection of chemical stimulation related to olfactory perception, olfactory perception, the positive regulation of transcription through RNA polymerase II, transcription regulation of DNA template, multicellular biological development, cell differentiation and phosphorylation, respectively. In addition, $18.05 \%$ of the target genes also participated in the oxidation-reduction process, protein transport, phosphorylation and ubiquitination, ion transport, the cell cycle and the regulation of apoptosis.

As demonstrated by the cellular component (CC) enrichment analysis, $90.91 \%, 63.42 \%$ and $58.69 \%$ of the target genes were involved in cell membrane composition, cytoplasmic composition, and plasma membrane composition, respectively. In addition, $52.03 \%, 35.28 \%$, and $28.85 \%$ of the target genes participated in the composition of the nucleus, cytoplasmic cells, and nucleoplasm, respectively.

The molecular function (MF) enrichment analysis demonstrated that $65.59 \%, 33.03 \%, 22.53 \%$ and $20.25 \%$ of the target genes participated in protein binding, metal ion binding, $\mathrm{G}$ protein-coupled receptor activity and transferase activity, respectively. In addition, some genes were found to be involved in the combination of nucleotides and ATP and DNA molecules. The results of the GO enrichment analysis are presented in Fig. 6.

\section{KEGG enrichment analysis}

The results of the KEGG enrichment analysis revealed that the target genes primarily participated in the modulatory control of human diseases, metabolism and biological systems. In addition, a large proportion of genes were classified into the categories of cellular process, environmental information processing and genetic information processing. In this section, the percentage of genes refers to the percentage of significantly enriched genes in the corresponding secondary categories.

As shown in Fig. 7A, most significantly enriched target genes (almost three-quarters of all enriched genes) were related to infectious diseases (associated with viral, parasitic and bacterial infections) and cancer in the category of human diseases. In addition, the percentage of genes in each secondary category exceeded 50\%. Within the metabolism category, 51 (54.26\%), 122 (47.84\%), 84 (47.73\%), 111 $(49.33 \%)$ and $92(53.18 \%)$ target genes were significantly enriched in nucleotide metabolism, lipid metabolism, amino acid metabolism, carbohydrate metabolism, and glycan biosynthesis and metabolism, respectively. The percentage of enriched genes in each secondary category related to organismal systems exceeded $40 \%$ as follows: ageing (56.06\%), sensory system (52.81\%), development and regeneration system (50.52\%), nervous system (50.3\%), endocrine system (49.82\%), environmental 
adaptation (46.11\%), circulatory system (48.08\%), immune system (49.28\%), digestive system (45.66\%), and excretory system (40.22\%). Within the environmental information processing category, 249 genes were predominantly enriched in pathways related to signalling molecule interactions, 585 genes were related to signal transduction, and 16 genes were related to membrane transduction. Moreover, within the genetic information processing category, 47 genes were enriched in nucleotide replication and repair, and 132 genes were enriched in protein folding, sorting and degradation. Strikingly, the data related to the cell process category revealed that there were 183 target genes predominantly gathered in modulating the control of cell growth and death. Moreover, the rich factor analysis (Fig. 7B) showed that these target genes were predominantly enriched in apoptosis, focal adhesion, neuroactive ligandreceptor interaction, p53 signalling pathway and hepatitis $\mathrm{B}$. This finding is crucial for our subsequent screening of the target genes involved in modulating apoptosis in macrophages infected with Mtb.

\section{Visualization analysis of the KEGG enrichment results}

Metascape (https://metascape.org) was selected to visualize and analyse the biological functions of the target genes enriched in cell growth and death regulation. A total of 183 genes were transformed into their corresponding M. musculus Entrez gene IDs, and related enrichment analyses were then performed. The top twenty clusters and their representative enriched terms were converted into a network layout.

As indicated in the bar graph shown in Fig. 8, apoptosis (ko04210), oocyte meiosis (ko04114), cell cycle (ko04110), hepatitis B (mmu05161) and p53 signalling (WP2902) were the top five significantly enriched terms across the input genes, and the specific gene annotation information of each cluster is summarized in Table 3. The result demonstrated that these genes were mainly involved in signalling, cellular processes and the regulation of metabolic and biological processes. The same result is displayed in Fig. 9, where each node stands for an enriched term, coloured first based on its cluster ID and then according to its $p$ value.

An extended protein-protein interaction network was framed, and 9 clusters were extracted using the MCODE algorithm. The pathways and biological processes enrichment analysis was utilized to each MCODE component independently, as shown in Fig. 10. Three optimal scoring terms were preserved as the functional description of the relevant components and highlighted in Table 4. The results demonstrated that the enriched genes were predominantly involved in regulating the cellular apoptosis process and oocyte meiosis. MCODE_1 and MCODE_3 were associated with the regulation of the cell cycle; MCODE_2 and MCODE_4 mostly participated in apoptosis pathways; MCODE_5 was associated with the longevity regulating pathway; and MCODE_6 was associated with an interleukin-6 family signalling pathway and $C D 4^{+}$or $C D 8^{+} \alpha-\beta$ T cell lineage commitment. As shown by the data presented in Table 5, apoptosis was the term with the best score obtained from the MCODE analysis.

\section{qRT-PCR validation}


The relative expression of miRNA in exosomes was detected by qRT-PCR to validate the accuracy of the microRNA-Seq results. Six biological samples (three per group) were prepared, and exosomes were isolated by ultracentrifugation. Eight miRNAs were randomly selected from the list of differentially expressed miRNAs, and their relative expression levels in each sample were detected and are presented in Fig. 11.

\section{Discussion}

In the present study, six miRNAs differentially expressed in exosomes derived from RAW264.7 cells infected with BCG were preliminarily screened. The bioinformatics and enrichment analysis demonstrated that these exosomal miRNAs and their target genes were predominantly involved in multiple biological processes and signal pathways related to cellular growth and death.

TB remains a fatal threat to global human health and safety and ranks first in terms of mortality rate among all infectious diseases, and no optimal treatment for the effective control of TB infection has been established. Mtb is a typical intracellular pathogen that causes TB infection and mainly infects macrophages, which serve as the initial sites of pathogen survival and replication; and this finding also provides a potential mechanism for controlling the activation of uninfected macrophages mediated by $T$ cells (16). Once a pathogen is phagocytosed by macrophages, the body activates the immune defence system to eliminate the pathogen. In contrast, to adapt to its new environment, Mycobacteria competitively manipulate the network of host cell miRNAs to facilitate their survival during the host-pathogen interaction process. MiRNAs constitute a class of noncoding small RNA molecules with 18-24 nucleotides that are associated with the posttranscriptional regulation of mRNA (17). Any change in the structure or expression level of miRNAs may seriously affect biological processes and lead to the occurrence of pathological conditions (18).

In contrast, Mycobacterium infection can also alter the host cell exosome content. Exosomes are a subpopulation of extracellular vesicles with a specific plasma membrane-enclosed structure with a diameter between 30 and $150 \mathrm{~nm}$ that can shuttle cell-specific cargo, including RNAs, proteins, lipids and DNA, to other cells both in the vicinity or at distant sites in the body. Due to their nanoscale diameter and excellent histocompatibility, exosomes have been identified as ideal natural biological carriers that play a pivotal role in information transmission and intercellular communication $(19,20)$. Exosomal miRNAs can also be transferred or transported to recipient cells and induce some biological reactions in these cells. More and more studies have indicated that exosomal miRNAs control regulate genetic expressions and different cell viability in vivo and in vitro $(10,21,22)$.

Macrophages constitute the front line of defence in innate immunity, and apoptosis plays a critical role in the clearance of intracellular pathogenic bacteria (23). However, Mycobacteria have the ability to hijack the metabolism and energy pathways of host cells and disrupt the autophagy and apoptosis processes of infected cells (24). An increasing number of studies have found that Mtb is capable of evading immune surveillance and promoting its retention and survival in macrophages by manipulating the host 
cell miRNA network (25-27). Some of the let-7 family members were reported that they play an important role in the immune response to TB infection $(28,29)$. Several accurately expressed miRNAs, such as hsamiR-144-3p, -142-3p and -23a-5p, have also been shown to be strongly associated with the immune response to TB infection. Moreover, miR-142-3p can suppress phagocytosis by perturbing neural WiskottAldrich protein syndrome in host cells (30). By targeting ATG4a, miR-144-3p can prevent the autophagy process activation and promote bacillus infection and survival in BCG infected RAW264.7 cells (31). In the course of Mtb infection, miR-23a-5p may influence the TLR2/MYD88/NF-KB pathway by targeting TLR2 to manipulate autophagy and Mtb persistence (32).

Thus far, most previous studies have attempted to reveal the complex mechanisms of hostpathogen interactions at the cellular miRNA level, but the detailed roles of miRNAs in TB infection remain poorly understood. Therefore, we explored the possible related mechanisms at the exosomal miRNA level and attempted to unravel the secretion of Mtb during immune evasion. Our study demonstrated that the exosomal miRNAs expression level from the cell culture medium of macrophages between the two groups showed significant differences. Twenty-seven differentially expressed miRNAs were screened from the comparison of the infection and non-infection group, and these included 20 upregulated and 7 downregulated miRNAs, respectively, in the infection group. Among them, mmu-miR-27b-3p, -93-5p, $-25-$ $3 p,-1198-5 p$, -let-7c-5p and -let-7a-5p were the top six significantly differentially upregulated miRNAs $(p<$ 0.05 ) and fit for the subsequent analysis.

It was demonstrated that majority of the target gene products were located in the membrane, cytoplasm, plasma membrane, nucleus, cytosol, nucleoplasm and mitochondrion; their molecular functions were mostly enriched in protein and metal ion binding, $G$ protein-coupled receptor activity, protein and metal ion binding and transferase activity; furthermore, the target genes were involved in multiple biological processes, including the $\mathrm{G}$ protein-coupled receptor signalling pathway, signal transduction, the response to stimulation, the detection of chemical stimulation related to olfactory perception and the positive modulation of transcription through RNA polymerase II.

The KEGG enrichment analysis demonstrated that the predicted target genes widely participated in the regulation of pathways related to the following categories, including human diseases, metabolism, cellular processes, environmental information processing, genetic information processing and organismal systems. The results of this analysis contains apoptosis, focal adhesion, neuroactive ligandreceptor interaction, the p53 signalling pathway, hepatitis B, EGFR tyrosine kinase inhibitor resistance, microRNAs in cancer, the TNF signalling pathway, endocrine resistance, mismatch repair and the PI3K-Akt signalling pathway $(p \otimes 0.05)$.

Mtb is a slow-growing facultative intracellular parasite. Macrophages are not only the first stop during the host invasion process of Mtb but also serve as a potential niche used by mycobacteria to form latent infections. The prognosis of macrophages infected with Mtb determines the fate of intracellular pathogens. We further focused our attention on the target genes which closely connected to the modulation of cell growth and death pathways. In total, 183 target genes were collected for further data 
mining and functional visualization analysis using the Metascape online tool. The analysis of the PPI network and MCODE displayed that these proteins were tightly related to the cell cycle, oocyte meiosis, Parkinson's disease, apoptosis, the longevity regulating pathway and interleukin- 6 signalling. It is apparent that apoptosis was the predominant term.

Similar findings were reported (33), Aplipoor et al infected human monocyte-derived macrophages with BCG and identified a complex set of exosomal miRNAs. The enrichment analysis illustrated that these miRNAs were primarily taken part in the regulation of pathways related to host metabolic progression, cell signalling and infectious diseases. The result suggested that metabolic pathways participating in protective immunity of the host are weakened to favour bacterial survival within macrophages, and this effect ultimately leads to the persistence of intracellular bacteria. In another study, the researchers further investigated three miRNAs (miR-1224, -484, and -425) that regulate metabolic pathways during TB infection (34). They found that the increase in the relative expression level of miR-1224 was higher than miR-484 and miR-425 expression in the BCG-infected group. They hypothesized that Mtb was capable of tolerating the hostile microenvironment by triggering and subverting (repatterning) host metabolic pathways, and as a result, Mtb escapes degradation by lysosomes and forms a lipid-rich niche that favours its survival. Previous studies have confirmed that the modification of lipid metabolism in host cells plays a key role in intracellular bacterial retention during TB infection $(35,36)$. MiR-1224 also participates in the modulation of lipid metabolism.

As a type of programmed death, apoptosis is one of the important mechanisms by which macrophages govern TB infection in the host innate immune defence. However, the mechanism through which Mycobacteria trigger macrophage apoptosis remains largely unknown. Some studies have partially uncovered the regulatory mechanisms underlying the interplay between miRNAs and the host macrophage immune response and apoptosis during TB infection (25). A microarray profiling survey revealed that the expression of miRNAs derived from $\mathrm{CD} 14^{+}$monocytes of active pulmonary TB patients was downregulated, and nothing but miR-20a-5p expression could be conversed after successful anti-tuberculosis therapy (37). Functional in vitro experiments investigating anti-tuberculosis infection manifested that the downregulation of miR-20a-5p could trigger apoptosis in macrophages by targeting JNK2 to eliminate intracellular Mycobacterium. In another study, it was showed that the overexpression of miR-20b-5p in Mtb-infected macrophages was able to reduce the cell viability and induce apoptosis; conversely, the inhibition of the expression of miR-20b-5p promoted cell viability and weakened macrophage apoptosis. Mcl-1 was one of the predicted targets of miR-20b-5p, and the downregulation of this miRNA could upregulate Mcl-1 expression to facilitate the intracellular survival of Mycobacteria (38).

Above all, we found twenty-seven differentially expressed exosomal miRNAs in exosomes derived from BCG infected RAW264.7 cells, their target genes were positively corelated with the regulation of apoptosis. This discovery provides relatively strong evidence revealing the mechanism through which Mycobacteria evade the immune system at the exosomal miRNA level. Furthermore, it also provides an important direction for the sbsequent screening of specific miRNAs to investigate the detailed mechanism of host-pathogen interactions during TB infection for our team. Eight miRNAs, namely, mmu- 
miR-27b-3p, -25-3p, -98-5p, -30a-3p, -194-5p, -5110, -let-7a-5p and -let-7c-5p, were randomly selected from the list of differentially expressed miRNAs to verify the accuracy of the high-throughput sequencing data by qRT-PCR. The relative expression of mmu-mir-27b-3p, -let-7c-5p, -25-3p, -98-5p, -let-7a-5p and -30a-3p in the infection group indicated a consistent trend with the previous sequencing results, but the relative expression levels of the rest two miRNAs were contrary to the results obtained by sequencing, which may be related to the two different methods used for the exosome isolation and exosomal RNA extraction. Overall, the relative expression levels of the miRNAs showed significant differences between the infection and non-infection group $(p<0.05)$, which indicated that the high-throughput sequencing results were relatively accurate.

Exosomes are an important area of research related to human diseases and infectious agents that has sparked interest in recent years. Because exosomes have the characteristics of biocarriers and possess information regarding the body's pathological state with respect to the immune system, they may be used as regulators of the immune response to activate the regulation of the host immune response and infection (39). In our study, high-throughput sequencing revealed that the miRNA expression profiles changed in exosomes derived from macrophages infected with BCG. The discovery of these differentially expressed miRNAs is conducive to our recognition of the complicated mechanism of host-pathogen interactions during the pathogenesis of TB at the exosomal miRNA level. Moreover, these exosomal miRNAs may serve as potential immunotherapy targets in the effective control of TB infection, but this hypothesis needs further study. However, our studies also have some deficiencies. First, mouse rather than human macrophages were used in our study; second, we used an avirulent BCG strain instead of pathogenic virulent or weak strains (H37Rv or H37Ra) for the infection stimulation, which could have had a certain impact on our final sequencing results. In addition, using specimens from patients with clinically confirmed TB and healthy volunteers in the sequencing analysis and validation of the results would be more clinically relevant and rigorous.

\section{Conclusion}

In summary, the mechanisms through which M. tuberculosis evades immune surveillance during TB infection remain complex. Our study provides relatively strong evidence to better understand the pathogenesis of TB from the perspective of exosomal miRNAs differentially expressed in host macrophages. The interplay between pathogens and host cells is an intricately linked event involving multiple factors, such as the Mycobacteria strain species and virulence, the duration of the infection stimulus, the status of macrophages and other immune cells, alterations in the miRNA expression profile and levels, the bacterial vesicles produced by pathogens and the exosomes secreted from host cells, which might influence the prognosis and outcomes of TB.

\section{Abbreviations}




\begin{tabular}{ll} 
TB: & tuberculosis \\
\hline BCG & Bacillus Calmette-Guérin \\
\hline MTBC & Mycobacterium tuberculosis complex \\
\hline Mtb & Mycobacterium tuberculosis \\
\hline DMEM & Dulbecco's modified Eagle's medium \\
\hline FBS & foetal bovine serum \\
\hline PBS & phosphate buffered saline \\
\hline GO & Gene Ontology \\
\hline KEGG & Kyoto Encyclopedia of Genes and Genomes \\
\hline MCODE & Molecular Complex Detection \\
\hline BLAST & the Basic Local Alignment Search Tool
\end{tabular}

\section{Declarations}

\section{Ethics approval and consent to participate}

This article does not contain any studies with human participants or animals performed by the authors. The experimental protocol was established, according to the ethical guidelines of the Helsinki Declaration and was approved by the medical research ethics review committee of General Hospital of Ningxia Medical University. Ethics number:2019-035. We confirm that all methods and experimental protocols were approved by Ningxia Medical University.

\section{Consent for publication}

Not applicable.

\section{Competing interests}

The authors declare that they have no competing interests.

\section{Author Contributions:}

Conceptualization, R.M. and Z.G.; methodology, X.Z.; software, W.Y.; validation, X.Z., W.Y. and Y.Z.; writing -original draft preparation, X.Z.; writing-review and editing, Z.G.; supervision, Z.G.; project administration, R.M. All authors have read and agreed to the published version of the manuscript.

\section{Funding}


This work was supported by the National Natural Science Foundation of China [Grant number 8196090244].

\section{Availability of data and materials}

The datasets generated during the current study are available in the Genome Sequence Archive (Genomics, Proteomics \& Bioinformatics 2021) in National Genomics Data Center (Nucleic Acids Res 2021), China National Center for Bioinformation / Beijing Institute of Genomics, Chinese Academy of Sciences (GSA: CRA006010) that are publicly accessible at https://ngdc.cncb.ac.cn/gsa/s/ITsJI588.

\section{Acknowledgements}

We are very grateful to Professor Zhao Wei, the director of the Echinococcosis Laboratory at Ningxia Medical University, for his invaluable technical suggestions and Li Gang, the director of the Ningxia Key Laboratory of Pathogenic Microorganisms, for providing the experimental site.

\section{References}

1. World Health Organization. Global Tuberculosis Report 2021. Geneva: World Health Organization; 20212021.

2. Liu CH, Liu H, Ge B. Innate immunity in tuberculosis: host defense vs pathogen evasion. Cell Mol Immunol. 2017;14(12):963-75.

3. Zhai W, Wu F, Zhang Y, Fu Y, Liu Z. The Immune escape mechanisms of mycobacterium tuberculosis. Int J Mol Sci. 2019;20(2).

4. Abdalla $A E$, Ejaz $H$, Mahjoob MO, Alameen AAM, Abosalif KOA, Elamir MYM, et al. Intelligent mechanisms of macrophage apoptosis subversion by mycobacterium. Pathogens. 2020;9(3):218.

5. Alipoor SD, Adcock IM, Tabarsi P, Folkerts G, Mortaz E. MiRNAs in tuberculosis: their decisive role in the fate of TB. Eur J Pharmacol. 2020;886:173529.

6. Alipoor SD, Tabarsi P, Varahram M, Movassaghi M, Dizaji MK, Folkerts G, et al. Serum exosomal miRNAs are associated with active pulmonary tuberculosis. Dis Markers. 2019b;2019:1907426.

7. Ha M, Kim VN. Regulation of microRNA biogenesis. Nat Rev Mol Cell Biol. 2014;15(8):509-24.

8. Kalluri R, LeBleu VS. The biology, function, and biomedical applications of exosomes. Science. 2020;367(6478):eaau6977.

9. Bettencourt P, Carmo N, Pires D, Timóteo P, Anes E. Mycobacterial infection of macrophages: the effect of the multiplicity of infection. In: Méndez-Vilas A, editor. Antimicrobial Research: Novel Bioknowledge and Educational Programs. Badajoz, Spain: Formatex Research Center; 2017. p. 65164.

10. Singh PP, Li L, Schorey JS. Exosomal RNA from Mycobacterium tuberculosis-Infected Cells Is Functional in Recipient Macrophages. Traffic. 2015;16(6):555-71. 
11. Szklarczyk D, Gable AL, Lyon D, Junge A, Wyder S, Huerta-Cepas J, et al. STRING v11: protein-protein association networks with increased coverage, supporting functional discovery in genome-wide experimental datasets. Nucleic Acids Res. 2019;47(D1):D607-D13.

12. Stark C, Breitkreutz BJ, Reguly T, Boucher L, Breitkreutz A, Tyers M. BioGRID: a general repository for interaction datasets. Nucleic Acids Res. 2006;34(Database issue):D535-9.

13. Li T, Wernersson R, Hansen RB, Horn H, Mercer J, Slodkowicz G, et al. A scored human protein-protein interaction network to catalyze genomic interpretation. Nat Methods. 2017;14(1):61-4.

14. Bader GD, Hogue CW. An automated method for finding molecular complexes in large protein interaction networks. BMC Bioinform. 2003;4:2.

15. Shannon P, Markiel A, Ozier O, Baliga NS, Wang JT, Ramage D, et al. Cytoscape: a software environment for integrated models of biomolecular interaction networks. Genome Res. 2003;13(11):2498-504.

16. Singh PP, Smith VL, Karakousis PC, Schorey JS. Exosomes isolated from mycobacteria-infected mice or cultured macrophages can recruit and activate immune cells in vitro and in vivo. J Immunol. 2012;189(2):777-85.

17. Bartel DP. MicroRNAs: genomics, biogenesis, mechanism, and function. Cell. 2004;116(2):281-97.

18. Alipoor SD, Adcock IM, Garssen J, Mortaz E, Varahram M, Mirsaeidi M, et al. The roles of miRNAs as potential biomarkers in lung diseases. Eur J Pharmacol. 2016;791:395-404.

19. Jadli AS, Ballasy N, Edalat P, Patel VB. Inside(sight) of tiny communicator: exosome biogenesis, secretion, and uptake. Molecular and cellular biochemistry. 2020;467(1-2):77-94.

20. O'Brien K, Breyne K, Ughetto S, Laurent LC, Breakefield XO. RNA delivery by extracellular vesicles in mammalian cells and its applications. Nat Rev MolCell Biol. 2020;21(10):585-606.

21. Chen WX, Liu XM, Lv MM, Chen L, Zhao JH, Zhong SL, et al. Exosomes from drug-resistant breast cancer cells transmit chemoresistance by a horizontal transfer of microRNAs. PloS one. 2014;9(4):e95240.

22. Ismail N, Wang Y, Dakhlallah D, Moldovan L, Agarwal K, Batte K, et al. Macrophage microvesicles induce macrophage differentiation and miR-223 transfer. Blood. 2013;121(6):984-95.

23. Behar SM, Martin CJ, Booty MG, Nishimura T, Zhao X, Gan HX, et al. Apoptosis is an innate defense function of macrophages against mycobacterium tuberculosis. Mucosal Immunol. 2011;4(3):27987.

24. Alipoor SD, Adcock IM, Folkerts G, Garssen J, Mortaz E. A bioinformatics analysis of exosomal microRNAs released following mycobacterial infection. Int J Mycobacteriol. 2019a;8(3):218-22.

25. Agarwal RG, Sharma P, Nyati KK. microRNAs in mycobacterial infection: modulation of host immune response and apoptotic pathways. Immune Netw. 2019;19(5):e30.

26. Kim JK, Kim TS, Basu J, Jo EK. MicroRNA in innate immunity and autophagy during mycobacterial infection. Cell Microbiol. 2017;19(1). 
27. Loeuillet C, Martinon F, Perez C, Munoz M, Thome M, Meylan PR. Mycobacterium tuberculosis subverts innate immunity to evade specific effectors. J Immunol. 2006;177(9):6245-55.

28. Fu Y, Yi Z, Wu X, Li J, Xu F. Circulating microRNAs in patients with active pulmonary tuberculosis. J Clin Microbiol. 2011;49(12):4246-51.

29. Sharbati J, Lewin A, Kutz-Lohroff B, Kamal E, Einspanier R, Sharbati S. Integrated microRNA-mRNAanalysis of human monocyte derived macrophages upon mycobacterium avium subsp. hominissuis infection. PLoS One. 2011;6(5):e20258.

30. Bettencourt P, Marion S, Pires D, Santos LF, Lastrucci C, Carmo N, et al. Actin-binding protein regulation by microRNAs as a novel microbial strategy to modulate phagocytosis by host cells: the case of N-Wasp and miR-142-3p. Front Cell Infect Microbiol. 2013;3:19.

31. Guo L, Zhou L, Gao Q, Zhang A, Wei J, Hong D, et al. MicroRNA-144-3p inhibits autophagy activation and enhances Bacillus Calmette-Guerin infection by targeting ATG4a in RAW264.7 macrophage cells. PloS one. 2017;12(6):e0179772.

32. Gu X, Gao Y, Mu DG, Fu EQ. MiR-23a-5p modulates mycobacterial survival and autophagy during mycobacterium tuberculosis infection through TLR2/MyD88/NF-kappaB pathway by targeting TLR2. Exp Cell Res. 2017;354(2):71-7.

33. Alipoor SD, Mortaz E, Tabarsi P, Farnia P, Mirsaeidi M, Garssen J, et al. Bovis bacillus calmette-guerin (BCG) infection induces exosomal miRNA release by human macrophages. J Transl Med. 2017;15(1):105.

34. Alipoor SD, Mortaz E, Tabarsi P, Marjani M, Varahram M, Folkerts G, et al. miR-1224 expression is increased in human macrophages after infection with bacillus calmette-guerin (BCG). Iran J Allergy Asthma Immunol. 2018;17(3):250-7.

35. Daniel J, Maamar H, Deb C, Sirakova TD, Kolattukudy PE. Mycobacterium tuberculosis uses host triacylglycerol to accumulate lipid droplets and acquires a dormancy-like phenotype in lipid-loaded macrophages. PLoS Pathog. 2011;7(6):e1002093.

36. Kim MJ, Wainwright HC, Locketz M, Bekker LG, Walther GB, Dittrich C, et al. Caseation of human tuberculosis granulomas correlates with elevated host lipid metabolism. EMBO Mol Med. 2010;2(7):258-74.

37. Zheng X, Ye C, Zhao J, Bian P, Zhang Y, Jia Z. [Alterations and clinical signifecance of exosomecontaining innate immunity related IncRNAs in patients of hemorrhagic fever with renal syndrome]. Xi bao yu fen zi mian yi xue za zhi = Chinese journal of cellular and molecular immunology. 2016;32(11):1522-6.

38. Zhang D, Yi Z, Fu Y. Downregulation of miR-20b-5p facilitates Mycobacterium tuberculosis survival in RAW 264.7 macrophages via attenuating the cell apoptosis by Mcl-1 upregulation. J Cell Biochem. 2019;120(4):5889-96.

39. Hadifar S, Fateh A, Yousefi MH, Siadat SD, Vaziri F. Exosomes in tuberculosis: Still terra incognita? J Cell Physiol. 2019;234(3):2104-11. 


\section{Tables}

Table 1. List of mmu-miRNA primer sequences.

\begin{tabular}{|c|c|c|}
\hline No. & mmu-miRNA & Primer sequence ( $\left(5^{\prime}\right.$ to $\left.3^{\prime}\right)$ \\
\hline \multirow[t]{2}{*}{1} & mmu-let-7a-5p & RT: CTCAACTGGTGTCGTGGAGTCGGCAATTCAGTTGAGAACTATAC \\
\hline & & F: ACACTCCAGCTGGGTGAGGTAGTAGGTTGT \\
\hline \multirow[t]{2}{*}{2} & mmu-let-7c-5p & RT: CTCAACTGGTGTCGTGGAGTCGGCAATTCAGTTGAGAACCATAC \\
\hline & & F: ACACTCCAGCTGGGTGAGGTAGTAGGTTGT \\
\hline \multirow[t]{2}{*}{3} & mmu-miR-25-3p & RT: CTCAACTGGTGTCGTGGAGTCGGCAATTCAGTTGAGTCAGACCG \\
\hline & & F: ACACTCCAGCTGGGCATTGCACTTGTCTCG \\
\hline \multirow[t]{2}{*}{4} & mmu-miR-27b-3p & RT: CTCAACTGGTGTCGTGGAGTCGGCAATTCAGTTGAGGCAGAACT \\
\hline & & F: ACACTCCAGCTGGGTTCACAGTGGCTAAG \\
\hline \multirow[t]{2}{*}{5} & mmu-miR-30a-3p & RT: CTCAACTGGTGTCGTGGAGTCGGCAATTCAGTTGAGGCTGCAAA \\
\hline & & F: ACACTCCAGCTGGGCTTTCAGTCGGATGTT \\
\hline \multirow[t]{2}{*}{6} & mmu-miR-98-5p & RT: CTCAACTGGTGTCGTGGAGTCGGCAATTCAGTTGAGAACAATAC \\
\hline & & F: ACACTCCAGCTGGGTGAGGTAGTAAGTTGT \\
\hline \multirow[t]{2}{*}{7} & mmu-miR-194-5p & RT: CTCAACTGGTGTCGTGGAGTCGGCAATTCAGTTGAGTCCACATG \\
\hline & & F: ACACTCCAGCTGGGTGTAACAGCAACTCCA \\
\hline \multirow[t]{2}{*}{8} & mmu-miR-5110 & RT: CTCAACTGGTGTCGTGGAGTCGGCAATTCAGTTGAGAATTCCAC \\
\hline & & F: ACACTCCAGCTGGGGGAGGAGGTAGAGGGTGGT \\
\hline \multirow[t]{2}{*}{9} & mmu-U6 & RT: CGAATTTGCGTGTCATCCTTG \\
\hline & & F: CTCGCTTCGGCAGCACATATAC \\
\hline
\end{tabular}

Table 2. Differentially expressed miRNAs and their expression levels. 


\begin{tabular}{|c|c|c|c|c|c|}
\hline miRNA & Fold change & $\log 2$ FC & $p$-value & Exp level & Up/down \\
\hline mmu-miR-27b-3p & 1.17 & 0.22 & $2.22 \mathrm{E}-02$ & high & up \\
\hline mmu-miR-93-5p & 1.19 & 0.26 & 2.84E-02 & high & up \\
\hline mmu-miR-25-3p & 1.33 & 0.41 & $3.98 \mathrm{E}-02$ & high & up \\
\hline mmu-miR-1198-5p & 1.16 & 0.21 & 4.19E-02 & high & up \\
\hline mmu-let-7c-5p & 1.28 & 0.36 & 4.36E-02 & high & up \\
\hline mmu-let-7a-5p & 1.28 & 0.36 & 4.36E-02 & high & up \\
\hline mmu-miR-7658-5p & inf & $\inf$ & $1.44 \mathrm{E}-02$ & low & up \\
\hline mmu-miR-7069-5p & inf & $\inf$ & $2.85 \mathrm{E}-02$ & low & up \\
\hline mmu-miR-8092 & inf & $\inf$ & 3.35E-02 & low & up \\
\hline mmu-miR-98-5p & 1.18 & 0.24 & 1.10E-02 & middle & up \\
\hline mmu-miR-212-3p & 1.38 & 0.47 & $1.36 \mathrm{E}-02$ & middle & up \\
\hline mmu-miR-181b-5p & 1.25 & 0.32 & $1.78 \mathrm{E}-02$ & middle & up \\
\hline mmu-miR-3057-5p & 1.40 & 0.49 & $2.00 \mathrm{E}-02$ & middle & up \\
\hline mmu-miR-203-3p & 2.55 & 1.35 & $2.23 \mathrm{E}-02$ & middle & up \\
\hline mmu-miR-6516-5p & 3.40 & 1.77 & $2.62 \mathrm{E}-02$ & middle & up \\
\hline mmu-miR-181d-5p & 1.97 & 0.97 & $3.38 \mathrm{E}-02$ & middle & up \\
\hline mmu-miR-30a-3p & 1.30 & 0.37 & $3.56 \mathrm{E}-02$ & middle & up \\
\hline mmu-miR-1933-3p & 2.03 & 1.02 & 3.77E-02 & middle & up \\
\hline mmu-miR-148b-5p & 1.70 & 0.76 & 3.80E-02 & middle & up \\
\hline mmu-miR-99b-3p & 1.34 & 0.42 & 4.88E-02 & middle & up \\
\hline mmu-mir-7018-p5 & 0.28 & -1.85 & $2.38 \mathrm{E}-02$ & middle & down \\
\hline mmu-miR-194-5p & 0.69 & -0.54 & 2.67E-02 & middle & down \\
\hline mmu-miR-301b-3p & 0.82 & -0.28 & 3.30E-02 & middle & down \\
\hline mmu-miR-5110 & 0.25 & -2.02 & $3.61 \mathrm{E}-02$ & middle & down \\
\hline mmu-miR-144-3p & 0.28 & -1.83 & $3.74 \mathrm{E}-02$ & middle & down \\
\hline mmu-miR-874-3p & 0.66 & -0.59 & 4.05E-02 & middle & down \\
\hline mmu-miR-363-3p & 0.34 & -1.54 & 4.92E-02 & middle & down \\
\hline
\end{tabular}

Table 3. Twenty major groups showing enhanced enrichment. 


\begin{tabular}{|c|c|c|c|c|c|c|}
\hline GO & Category & Description & Count & $\%$ & $\begin{array}{l}\log 10 \\
(P)\end{array}$ & $\log 10(q)$ \\
\hline ko04210 & $\begin{array}{l}\text { KEGG } \\
\text { pathway }\end{array}$ & Apoptosis & 51 & 27.87 & -70.51 & -66.23 \\
\hline ko04114 & $\begin{array}{l}\text { KEGG } \\
\text { pathway }\end{array}$ & Oocyte meiosis & 37 & 20.22 & -47.57 & -43.76 \\
\hline ko04110 & $\begin{array}{l}\text { KEGG } \\
\text { pathway }\end{array}$ & Cell cycle & 37 & 20.22 & -46.32 & -42.64 \\
\hline mmu05161 & $\begin{array}{l}\text { KEGG } \\
\text { pathway }\end{array}$ & Hepatitis B & 38 & 20.77 & -44.52 & -41.08 \\
\hline WP2902 & WikiPathways & p53 signalling & 28 & 15.30 & -39.87 & -36.63 \\
\hline G0:0097190 & GO BP & $\begin{array}{l}\text { Apoptotic signalling } \\
\text { pathway }\end{array}$ & 52 & 28.42 & -35.54 & -32.46 \\
\hline GO:1903047 & GO BP & Mitotic cell cycle process & 53 & 28.96 & -34.08 & -31.02 \\
\hline ko05166 & $\begin{array}{l}\text { KEGG } \\
\text { pathway }\end{array}$ & HTLV-I infection & 36 & 19.67 & -30.68 & -27.83 \\
\hline G0:0070997 & GO BP & Neuron death & 39 & 21.31 & -27.54 & -24.82 \\
\hline ko04380 & $\begin{array}{l}\text { KEGG } \\
\text { pathway }\end{array}$ & Osteoclast differentiation & 26 & 14.21 & -27.41 & -24.69 \\
\hline ko04621 & $\begin{array}{l}\text { KEGG } \\
\text { pathway }\end{array}$ & $\begin{array}{l}\text { NOD-like receptor } \\
\text { signalling pathway }\end{array}$ & 27 & 14.75 & -25.68 & -23.10 \\
\hline ko05169 & $\begin{array}{l}\text { KEGG } \\
\text { pathway }\end{array}$ & $\begin{array}{l}\text { Epstein-Barr virus } \\
\text { infection }\end{array}$ & 29 & 15.85 & -25.22 & -22.66 \\
\hline $\begin{array}{l}\text { R-MMU- } \\
3700989\end{array}$ & Reactome $G S$ & $\begin{array}{l}\text { Transcriptional regulation } \\
\text { by TP53 }\end{array}$ & 28 & 15.30 & -20.80 & -18.48 \\
\hline WP373 & WikiPathways & IL-3 signalling pathway & 19 & 10.38 & -19.72 & -17.46 \\
\hline ko05162 & $\begin{array}{l}\text { KEGG } \\
\text { pathway }\end{array}$ & Measles & 21 & 11.48 & -19.68 & -17.43 \\
\hline $\begin{array}{l}\text { R-MMU- } \\
1280218\end{array}$ & Reactome GS & Adaptive immune system & 37 & 20.22 & -19.23 & -17.01 \\
\hline GO:0006974 & GO BP & $\begin{array}{l}\text { Cellular response to DNA } \\
\text { damage stimulus }\end{array}$ & 39 & 21.31 & -18.33 & -16.14 \\
\hline G0:0031329 & GO BP & $\begin{array}{l}\text { Regulation of cellular } \\
\text { catabolic process }\end{array}$ & 38 & 20.77 & -17.85 & -15.69 \\
\hline $\begin{array}{l}\text { R-MMU- } \\
5218859\end{array}$ & Reactome $G S$ & Regulated necrosis & 14 & 7.65 & -17.59 & -15.44 \\
\hline GO:0018105 & GO BP & $\begin{array}{l}\text { Peptidyl-serine } \\
\text { phosphorylation }\end{array}$ & 27 & 14.75 & -17.53 & -15.40 \\
\hline
\end{tabular}


"Count" is the number of genes in the lists annotated with a given ontology term. "\%" is the percentage of all genes annotated with a given ontology term (only input genes annotated with at least one ontology term were included in the calculation). "Log10(P)" is the log base 10-transformed $p$-value. "Log10(q)" is the log base 10-transformed multitest adjusted $p$-value. BP:Biological process; GS: Gene sets.

Table 4. Six MCODE component annotation details.

\begin{tabular}{|c|c|c|c|c|}
\hline Colour & MCODE & Go & Description & $\log 10(P)$ \\
\hline & MCODE_1 & ko04110 & Cell cycle & -24.2 \\
\hline & MCODE_1 & mmu04110 & Cell cycle & -24.0 \\
\hline & MCODE_1 & ko04114 & Oocyte meiosis & -20.1 \\
\hline & MCODE_2 & WP3638 & Parkinson's disease & -13.5 \\
\hline & MCODE_2 & ko04210 & Apoptosis & -13.3 \\
\hline & MCODE_2 & $\mathrm{mmu} 04210$ & Apoptosis & -13.2 \\
\hline & MCODE_3 & ko04110 & Cell cycle & -26.9 \\
\hline & MCODE_3 & $\mathrm{mmu} 04110$ & Cell cycle & -26.7 \\
\hline & MCODE_3 & R-MMU-69278 & Cell cycle, mitotic & -22.4 \\
\hline & MCODE_4 & ko04210 & Apoptosis & -14.8 \\
\hline & MCODE_4 & $\mathrm{mmu} 04210$ & Apoptosis & -14.8 \\
\hline & MCODE_4 & ko04140 & Autophagy - animal & -12.9 \\
\hline & MCODE_5 & ko04213 & Longevity regulating pathway - multiple species & -17.7 \\
\hline & MCODE_5 & $\mathrm{mmu} 04213$ & Longevity regulating pathway - multiple species & -17.7 \\
\hline & MCODE_5 & $\mathrm{mmu} 04211$ & Longevity regulating pathway & -16.2 \\
\hline & MCODE_6 & R-MMU-1059683 & Interleukin- 6 signalling & -8.5 \\
\hline & MCODE_6 & R-MMU-6783589 & Interleukin- 6 family signalling & -7.4 \\
\hline & MCODE_6 & GO:0043369 & $\mathrm{CD} 4+$ or $\mathrm{CD} 8+$, alpha-beta $\mathrm{T}$ cell lineage commitment & -7.1 \\
\hline
\end{tabular}

If the network contained between 3 and 500 proteins, the Molecular Complex Detection (MCODE) algorithm was applied to identify densely connected network components.

Table 5. Top three MCODE terms. 


\begin{tabular}{|lll|}
\hline GO & Description & Log10(P) \\
\hline ko04210 & Apoptosis & -71.5 \\
\hline mmu04210 & Apoptosis & -70.9 \\
\hline ko04114 & Oocyte meiosis & -48.3 \\
\hline
\end{tabular}

A GO enrichment analysis of each MCODE network was performed to assign "meanings" to the network components, and only the top three terms based on the $p$-value were retained.

\section{Figures}

Figure 1

Characterization of exosomes by transmission electron microscopy (TEM). The exosome morphology was scanned by TEM. The scale bars represent $1 \mu \mathrm{m}$ (A), $500 \mathrm{~nm}$ (B), $200 \mathrm{~nm}$ (C), and $100 \mathrm{~nm}$ (D).

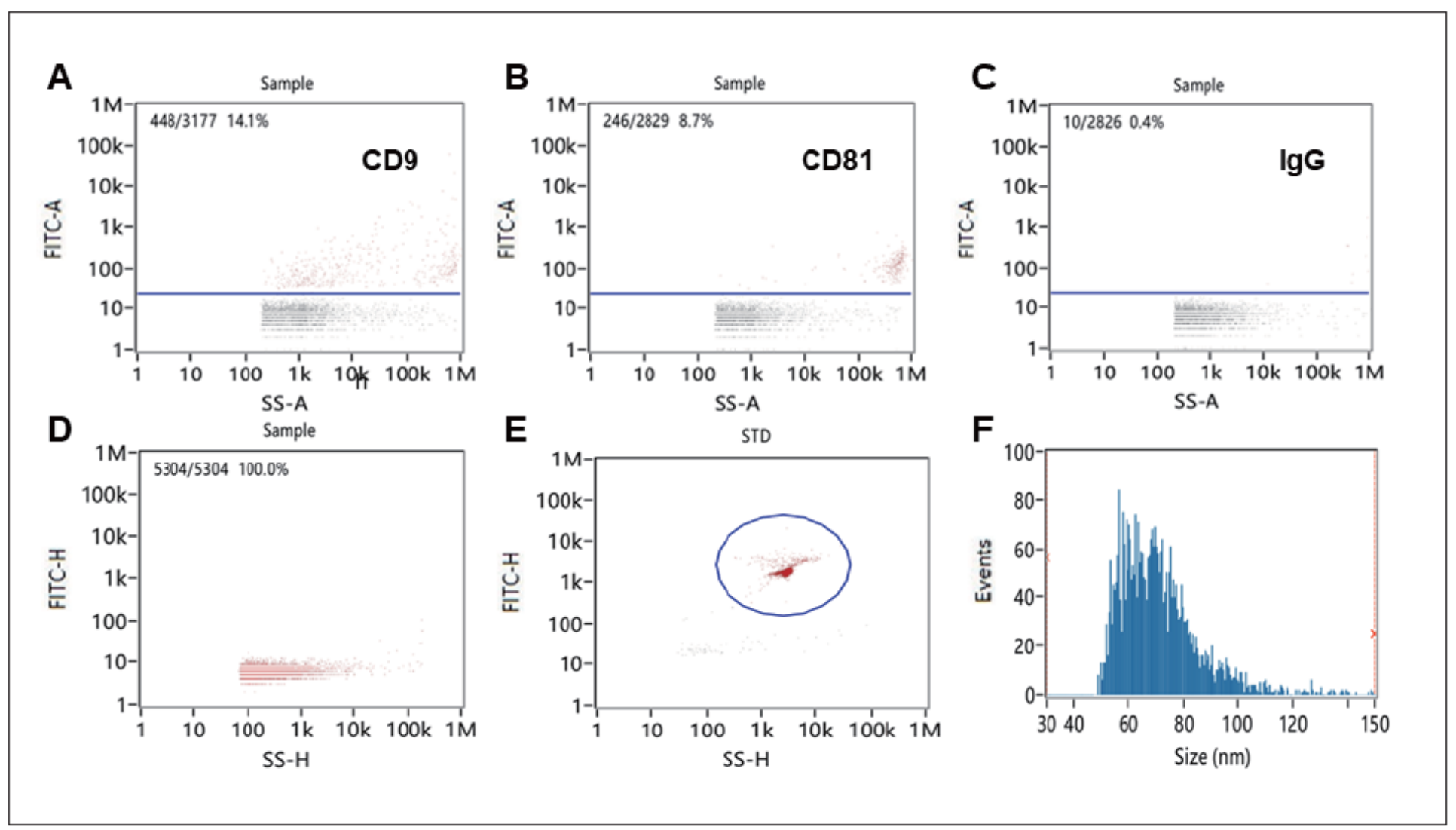

Figure 2

Characterization of exosomes by NanoFCM. The exosome transmembrane proteins CD9 and CD81 and isotype control FITC-IgG were detected and quantified by NanoFCM (A-C). The particle concentration was 
analysed by NanoFCM (D, E). The size distribution was analysed by NanoFCM (F).

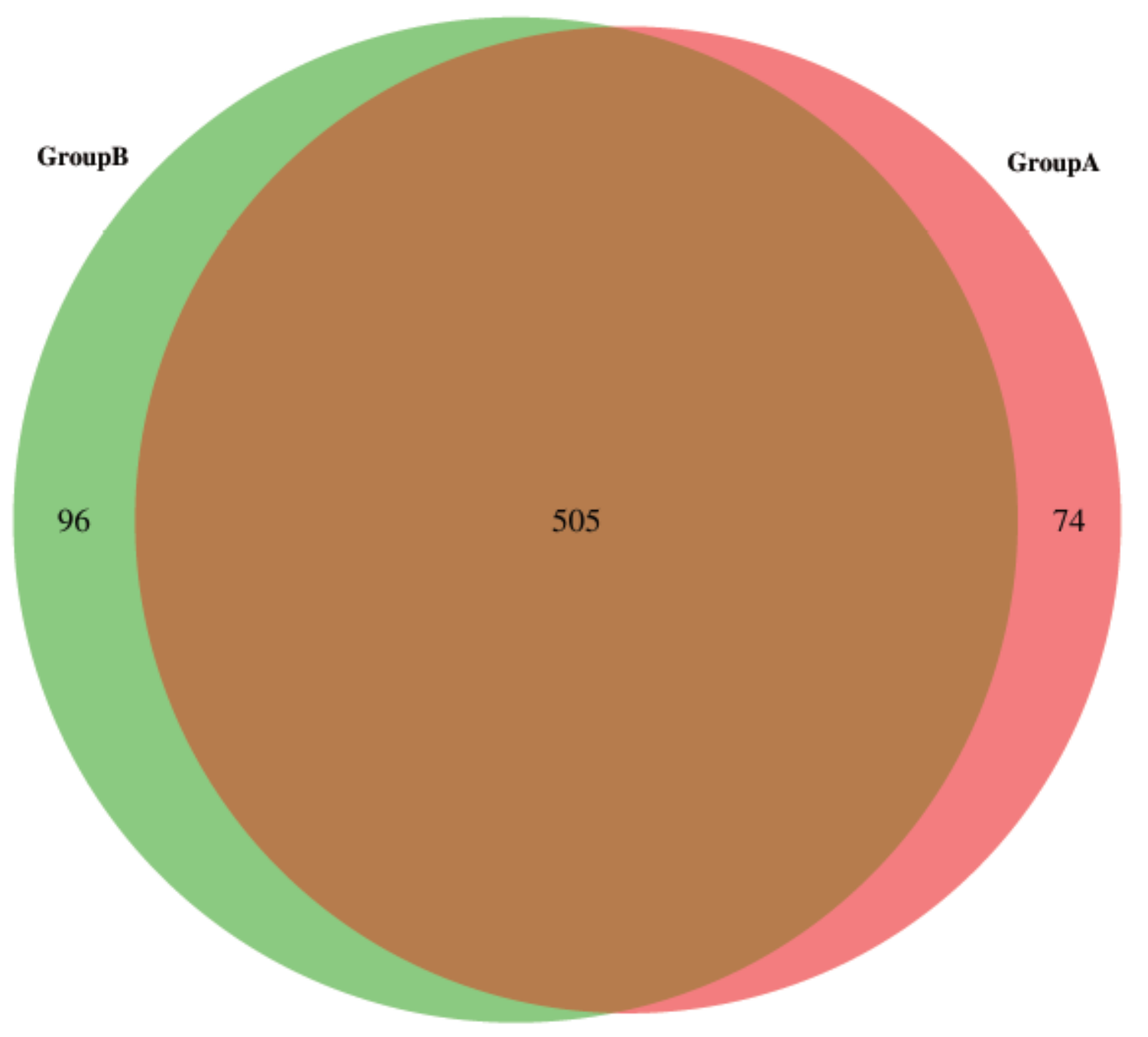

Figure 3

MiRNAs showing differential expression between the following two groups: Group A (noninfection group) and Group B (infection group). 


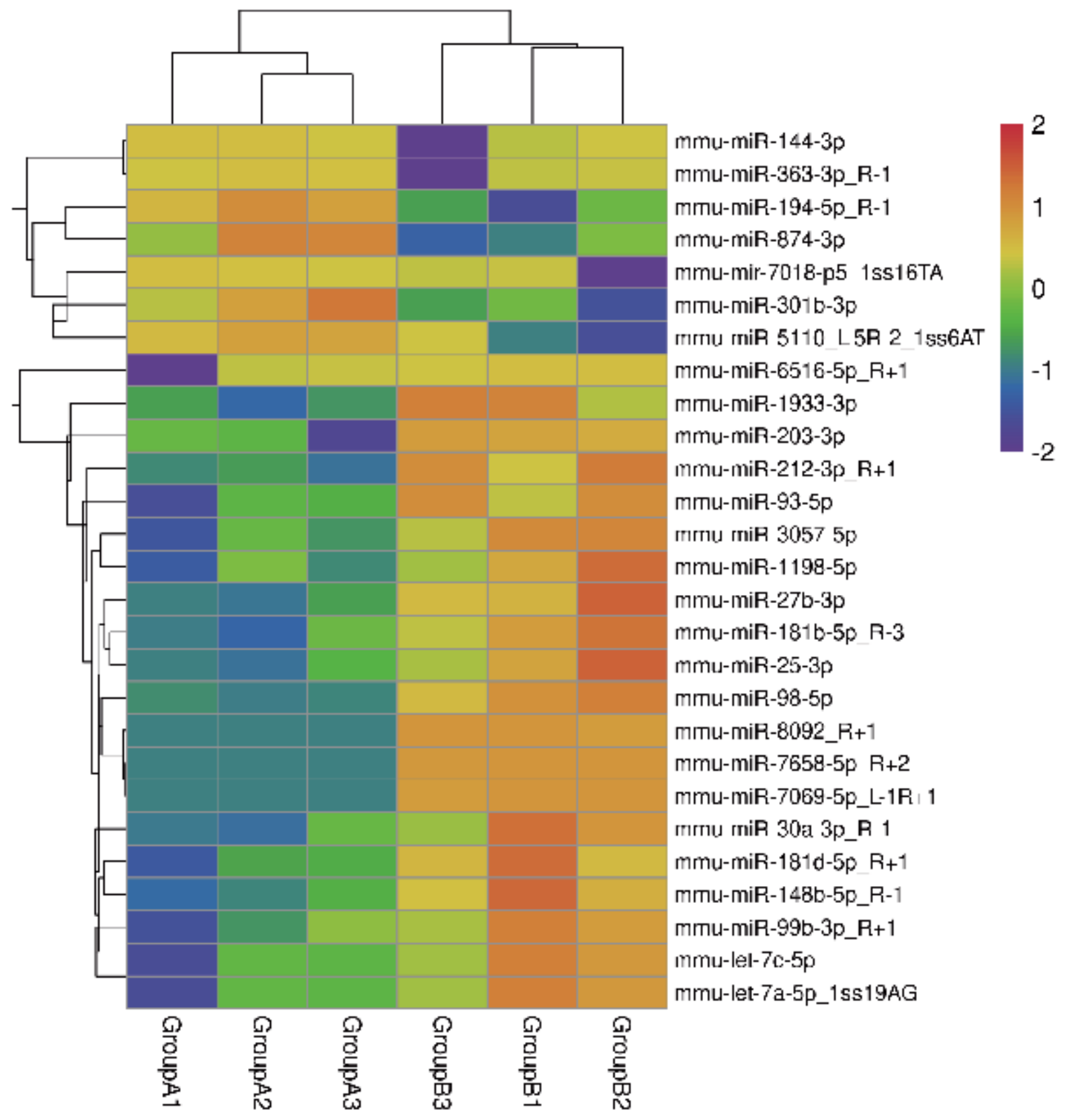

Figure 4

Heatmap of differently expressed miRNAs. The miRNA expression levels are presented as the $\log 10-$ transformed normalized values. The different colours represent different miRNA expression levels. The colour range from blue to orange represents an expression level range from low to high; specifically, the dark blue colour indicates high expression, and the orange colour indicates low expression. 


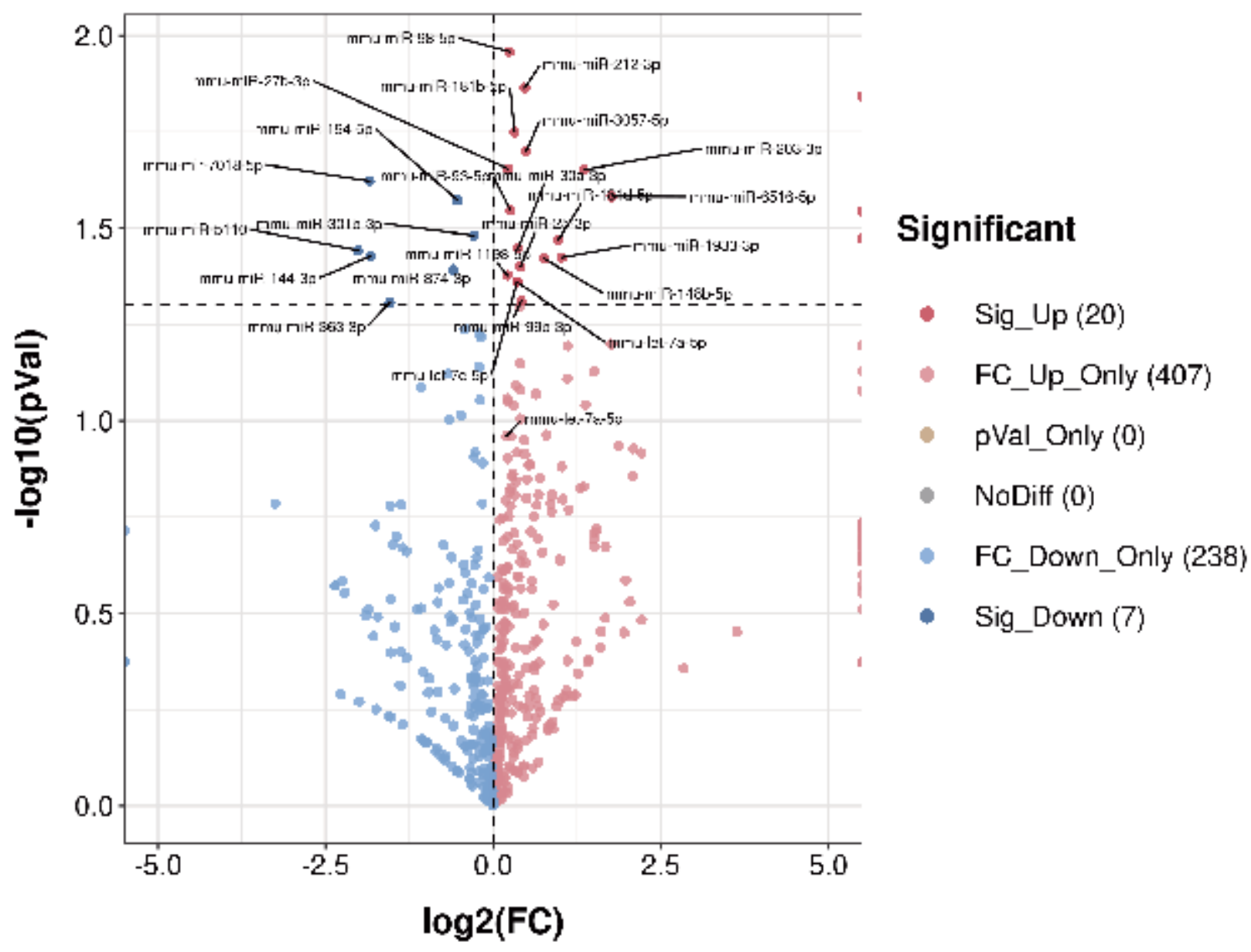

Figure 5

Volcano plot of differently expressed miRNAs. Log2(FC) represents the change in the differential expression of miRNAs in different samples; - $\log 10$ ( $p$ value) represents the statistical significance of the difference in miRNA expression; the red colour indicates significantly differentially upregulated genes, the blue colour indicates significantly differentially downregulated genes, and the grey points represent genes showing nonsignificant differential expression. 
A

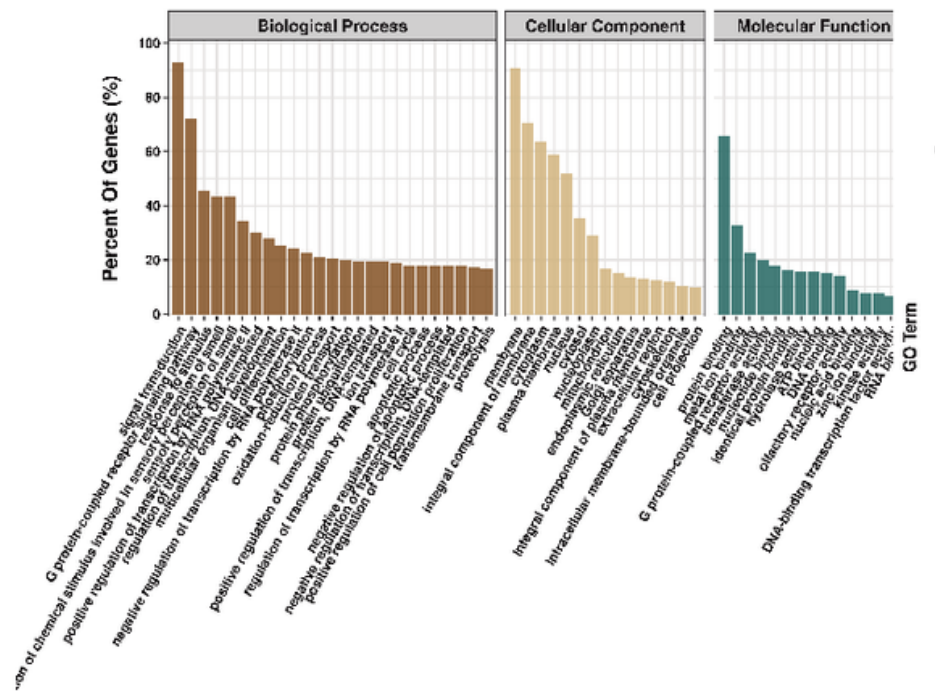

B

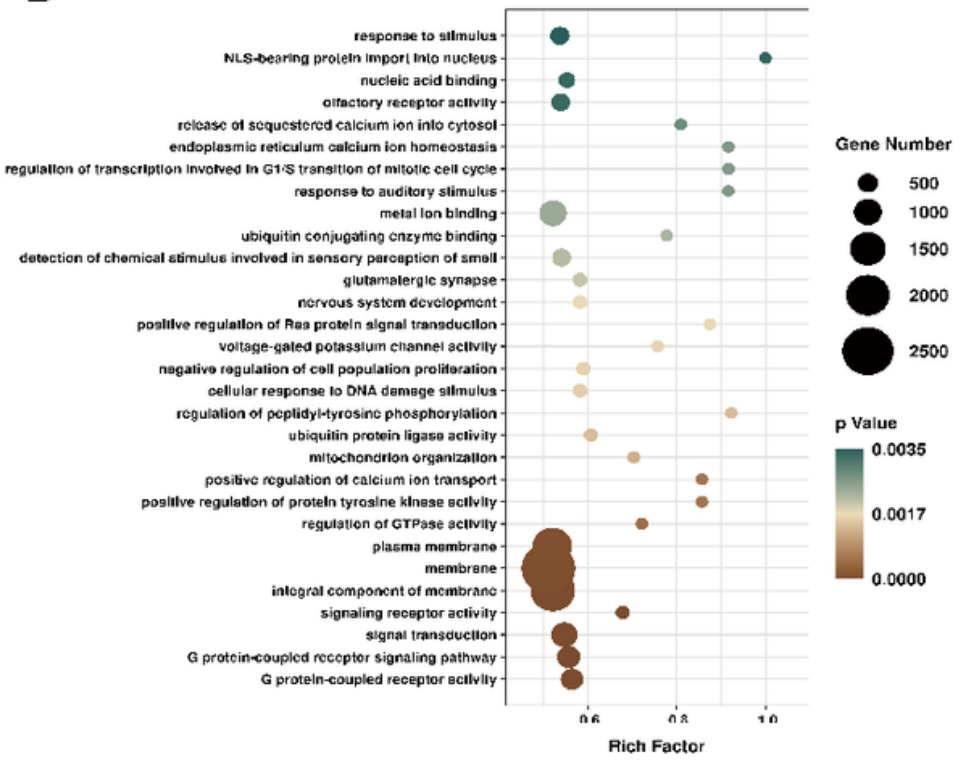

Figure 6

GO enrichment of the target genes. (A) Bar plot. (B) Scatterplot. The rich factor indicates the ratio of the number of differentially expressed genes divided by the total number of genes annotated with a specific term. For a term, a smaller $p$-value indicates a higher degree of enrichment. The diameter of the point represents the number of genes enriched with the item.

\section{Figure 7}

KEGG enrichment of the target genes. (A) Bar plot. (B) Scatterplot. The percentage of genes refers to the percentage of significantly enriched genes in the corresponding secondary categories. 


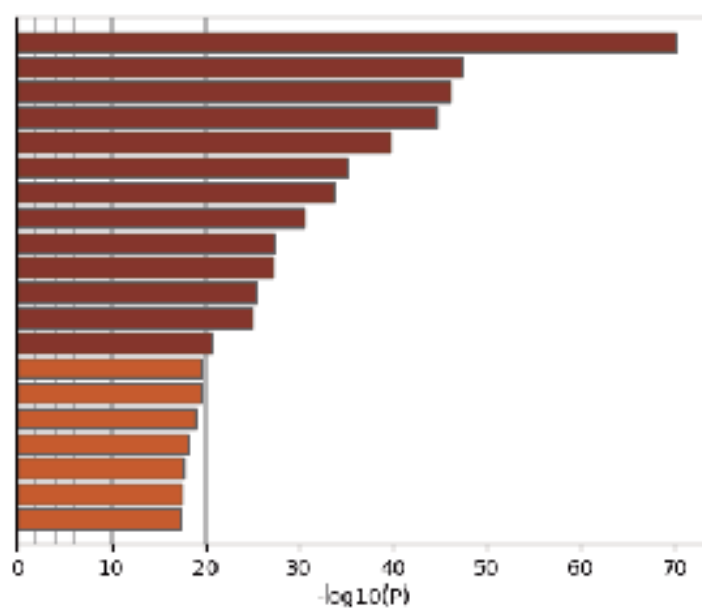

kec14210: Apraptosis

kaO4114: Opcyle meidsis

ko0 4110: Cel cycle

mmuob16l: Hepatitis $\mathrm{B}$

WP27907: p1s3 signaling

G0:0097 190: apoptetic signaling pathway

G0:1903047: m totic cell cycle process

k005166: HTLV-I Infectuon

GO-0070gazi nouran death

ke0439\%: Osterchal dillerentiat cen

kc04621: NOD-like receptor signalıng patwmay

kocs 169: Epstcin Barr virus infoction

R-MIAL-3 roDegay: Transcriptional Regulation by TPS.3

WP373: IL-3 signaling pathway

ko05162: Neasles

R-MIAU-1280218: /Doptive Immune system

GO:0006974: cellular response to UNA damoce stimulus

GO:0031379: tegulation ef terlular cat nbelic protess

P-MIMU-5218859: Regu ated Necrosis

G0:0018103: Dectidy-serine ohosonorylation

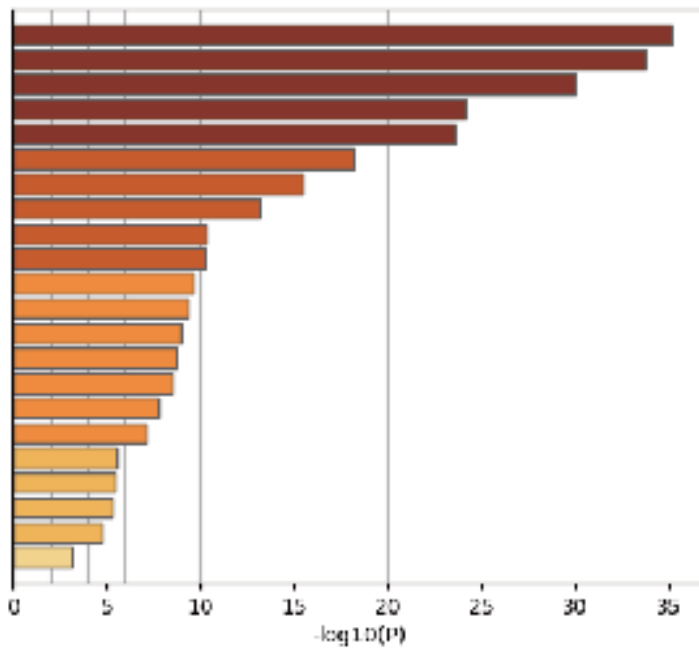

GO:00230s2: signalinte

Go:0009987: tellular procers

G0:0048518: postive regu aton of bucl opical process

G0.0000152: metalsol c process

G0:0050789: regulation of biolocica l process

GOodsagge: wosporse ta slimulas

G0:0048519: negalove regulalicin al bialogical packess

G0:00nsoc: bioloyica remulaticat

G0:0040011: Iscomotion

GO:000237e: immune syatem process

Go:ous11y: Iscalization

G0:0032s02: dizuelapriental proctes:

G0:0022414: reprotistive promers

G0:0040007: growth

GO-0044415; biclecical procsss involvod in interseceics interoction between organisms

G0:0048511: rhrthmic process

GO0032501: multicel lular arqanismal protess

G0:0016032: vial privess

G0:0051704: muit|-organism process

G0:0007610: behavio?

GO:0022510: bieleçical adhesion

GO0004a4a: biolorical phase

\section{Figure 8}

Bar graph of enriched terms across input gene lists (coloured by $p$-values). (A). Top 20 enriched terms across input gene lists. (B). Top Gene Ontology biological process terms. 
A

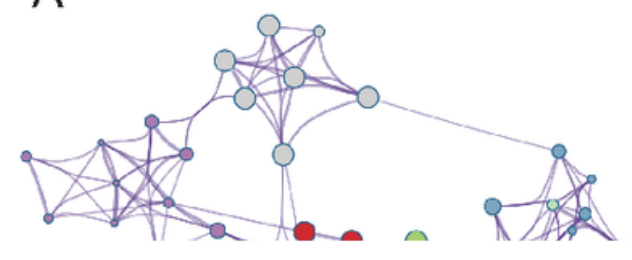

B

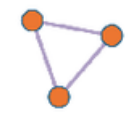

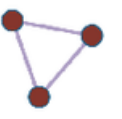

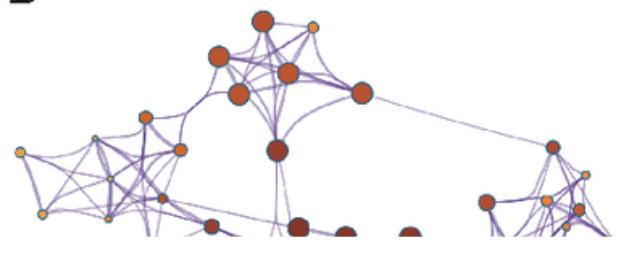

\section{Figure 9}

Network of enriched terms. (A) Coloured by cluster ID. Nodes that share the same cluster ID are typically close to each other. Terms with similarity scores $>0.3$ are linked by edges (the thickness of the edges represents the similarity score). (B) Coloured by the $p$-values. Terms containing more genes tended to have more significant $p$-values. A darker colour indicates that the node is more statistically significant.

A

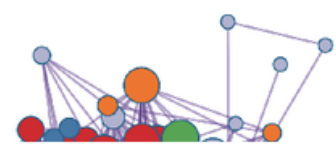

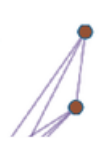
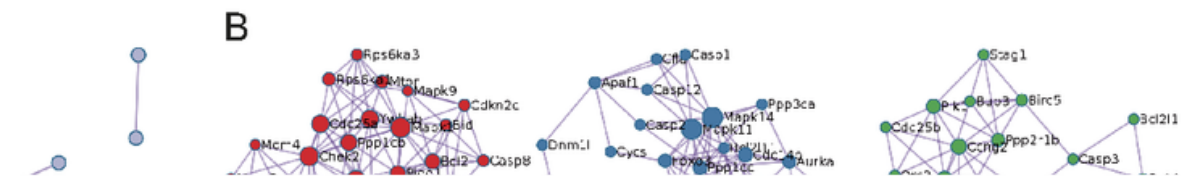

\section{Figure 10}

Protein-protein interaction enrichment analysis. Protein-protein interaction network and MCODE components identified in the gene lists. The MCODE algorithm was applied to the network to identify the 
neighbourhoods of protein-intensive connections. Each MCODE network is assigned a unique colour. MCODE: Molecular Complex Detection.
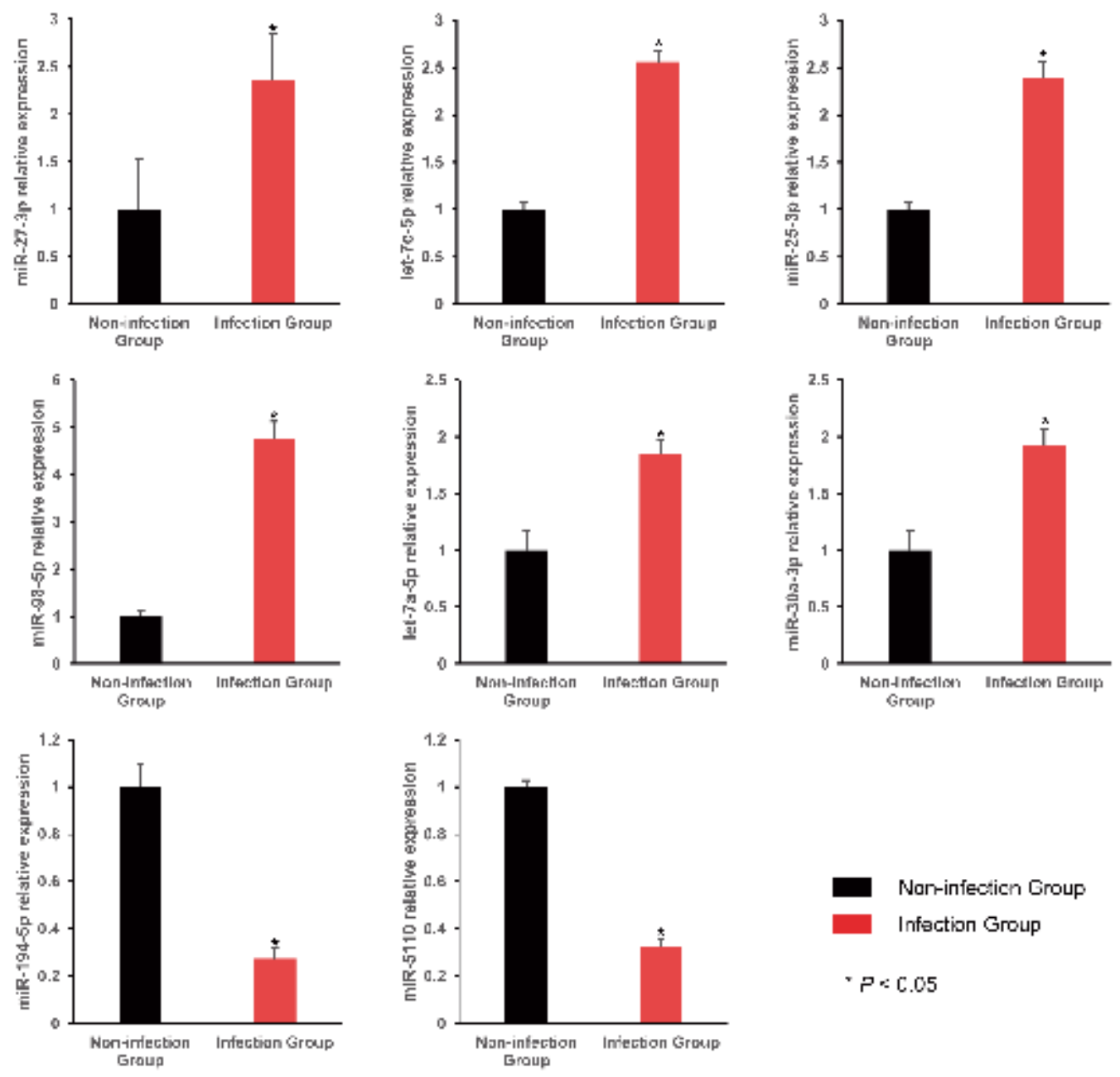

Figure 11

Validation of relative miRNA expression in exosomes by qRT-PCR. A1-A3: Noninfection group. B1-B3: Infection group 